\title{
A Quantitative Study on How Internet Search Ads Generate Consumer Traffic to Advertisers' Website
}

\author{
Jungsun Son*
}

\author{
ARTICLE INFO \\ Article history: \\ Received 21 March 2011 \\ Revised 20 May 2011 \\ Accepted 10 June 2011 \\ Keywords: \\ Internet Search Ad, \\ Advertising Website, \\ Ad Rank, \\ Click-Through Rate, \\ Advertising Message, \\ Consumer Traffic
}

\section{Introduction}

Internet advertising is helping companies' marketing efforts as a new medium capable of interactive communication, and the companies consider internet advertising a very important marketing means. Therefore, companies construct their websites for many internet users to access via the internet and are trying to promote their businesses to maximum effect.

As of June 2007, Korean internet users reached $75.5 \%$ of the total population $(3,443$ million people); reflecting this trend, the Korean internet advertising market recorded 8,907 billion won (about $\$ 8,907$ million), a growth rate of $34.5 \%$ in 2006 . The world's internet advertising market is expected to overtake the radio advertising market as 8.6\% share ad in 2009 (Korea Internet Marketing Association, 2006).

In particular, search ads began in 2002, which means that the ad is seen only when users search with keywords advertisers desired. Since 2005, search ads grew rapidly and surpassed banner ads.

* Chapel Hill, NC27514 (jsson85@gmail.com) International Journal of Knowledge Content Development \& Technology, 1(1): 7-24, 2011. [http://dx.doi.org/10.5865/IJKCT.2011.1.1.007] 
In 2010 the ads market is expected to exceed 1 trillion won (about $\$ 1$ billion). In recent years, new trends such as branding through keyword, cross-media ads with offline media, and the scientific process of search ads has provided the driving force for the growth of search ads.

As such, because internet search advertising has a lot of growth potential, researchers began studying them in earnest in 2002, and many research papers on search ads have been published. Lee, Lee, and Jin (2003) researched the search advertising techniques and technical aspects of implementation research. Kim, Kim, and Kim (2002) studied whether or not the location and exposure degree of ads can impact ad effectiveness. In addition, there were even attempts to present specific industry advertising strategies through analyzing literature reviews (Lim, 2002; Jeoung, 2006). Studies were carried out for verifying if the effectiveness of search ads depends on forms of expression and the type of searching ads (Kim, 2003; Song, 2005). The research into how the involvement theory works to the effectiveness of search ads was actively performed (Lee \& Choi, 2002).

However, as all the leading researchers indicated as a limitation, previous studies couldn't verify the response of consumers who found the search ads in an actual internet environment. Most of the studies were about demonstrating the effectiveness of search ads by using survey methods, and some studies presented the experimental model based on a hypothetical scenario. Therefore, there could be differences between statistical data and actual situations because the subjects were aware of the purpose of the study in advance. In addition, until now there has been no complex and numerical analysis research for verifying to what degree types of advertising messages and ranking influence the effectiveness of search ads when search ads were exposed to users.

This study proposed to verify the effectiveness of search ads based on the actual case data when the ads' consumers, connected to a portal site, visit the companies' website through search ads, overcoming the limitations of previous studies by doing so. First, this study analyzed how the type of advertising messages and search ads ranks, which are exposed to the consumers, affect the consumers' visiting the companies' websites by analyzing the actual consumer reaction. Second, this study proposed the most efficient model for search ads by creating a matrix based on the analysis of the detailed quantitative case data.

\section{Theoretical Background}

\subsection{The relationship between the involvement and the click rate}

The consumers' reaction when viewing advertising messages for the same products may appear differently. Likewise, the degree of personal importance and relevance or interest for any product or issue under certain circumstances is called the involvement (Lee, 2003).

The model which shows that the involvement has impact on the information processing and the attitude change of the consumers is Elaboration Likelihood Model (ELM) (Petty \& Cacioppo, 1986). According to this model, consumer attitudes are formed by two kinds of paths, the center path and the near path. The consumer processes information through the center path if the involvement is high, and the consumer processes information through the near path if the involvement is low. 
The concept of involvement started in the field of social psychology and has been used for analyzing the behavior of consumers. It is also usefully applied to the internet advertising.

When experimental research on internet banner ads and involvement was conducted, this study reported that a high involvement had formed the positive or negative attitudes for product items, depending on the information of banner ads exposing through the center path. On the other hand, it was explained that the consumer tends to click the banner based on the peripheral clues such as the shape, size, color of banner or the relevance of website etc. if the involvement is low (Kim, 2003).

According to Kim and Kim's study (2003), if a consumer's involvement with a website is higher, then the positive attitudes toward internet ads, the brand, and the purchase are also increased.

The attitude towards internet advertising, brand attitudes, and purchase intentions also improved when a consumer's involvement for websites is higher (Kim \& Kim, 2003). Only when the involvement is high and the feelings are positive did the website show a higher CTR (click-through rate), if the consumer was exposed to the banner ads and recognized them unconsciously (Kim \& Kim, 2003).

The impact of involvement appears differently depending on the nature of websites. The product advertising of high involvement is positive in the portal sites; on the other hand the product advertising of low involvement is positive in the community sites (Kim \& Lee, 2001).

Information retrieval on the internet can be classified as goal-oriented navigation and non-goal-oriented navigation depending on internet users' involvement (Hoffman \& Novak, 1996). The goal-oriented navigation is the situation of high involvement; it is activated when there is a specific target the consumer wanted. On the other hand, non-goal-oriented navigation is the situation of low involvement; it is activated when there is a lack of motivation, desire, or knowledge.

A consumer's involvement may appear differently depending on the type of internet advertising. Currently, the search ads and banner ads, which are representative of internet ads, can also be classified as the consumers' involvement. If consumer want to get the information in the advertiser's website by entering their wanted keyword and clicking the search ads, it is the situation of high involvement that is conducting a goal-oriented navigation. If consumer clicks the sensual banner advertising on the website, it's the situation of low involvement that is conducting a non-goal-oriented navigation.

In recent years, researchers have been observing the consumer's involvement in terms of visual aspects by the scientific method of eye-tracking. By using eye-tracking, we can measure directly what consumers give visual attention to. Eye-tracking can measure the visual attention by measuring immediate physiological responses such as the size and location of eyes, eye fixation and eye saccade. Because the data collected from eye-tracking has not been affected by the deformation or distortion that can occur after surveying, it is often more reliable data (Kim et al., 2006).

Jordan (2007) of 'Marketingpiligrim.com', citing a recent study by Jakob Nielsen, reported about web users' search type. According to his remarks, eye-tracking can show how web users' visual attention is being focused depending on the type of advertising, such as high involvement, which has a specific purpose. As shown in the Figure 1, a consumer's visual attention is not concentrated in sensual banner advertising, but in textual forms of advertising. In this experiment, the sections where many web users' attention stays were to be displayed in order of red $\rightarrow$ yellow $\rightarrow$ green. As a result, it was shown that most of users' attention was focused on the text conveying information, 
but not at banner advertising marked with rectangles. Jakob claimed that one needs to give an advertisement in textual form to be effective, meaning web users click their ads often.

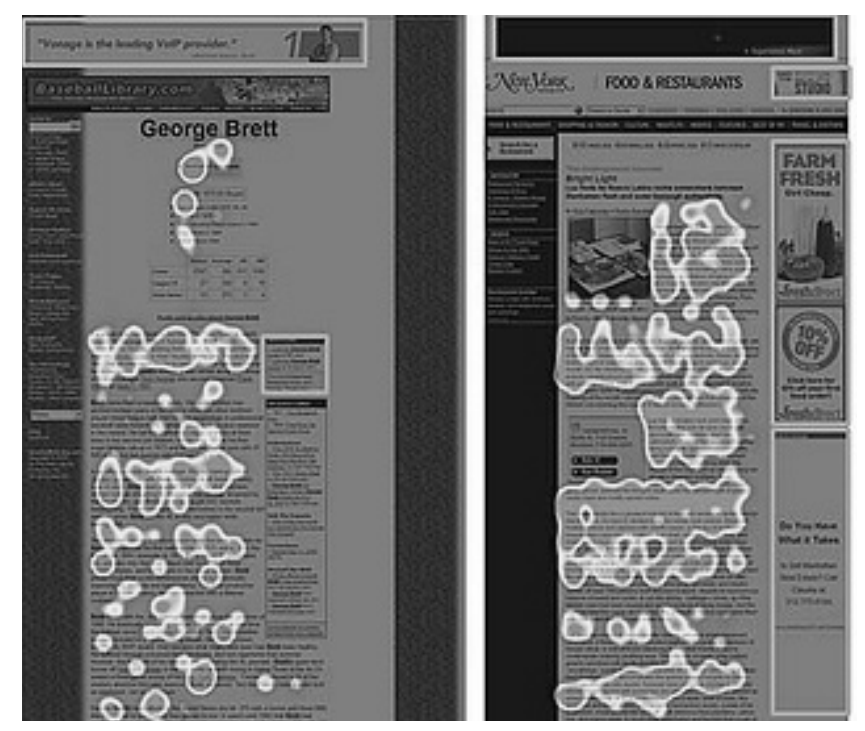

Fig. 1. Eye-tracking results on a webpage

A Korean survey study has also supported the previous research. KoreanClick conducted the survey on online search advertising, and according to the results, about $59 \%$ of users are aware of the advertising and about $82 \%$ of them clicked the ads (KoreanClick, 2005). This showed that consumers have very high involvement with the ads expressed in the form of text.

\subsection{The relationship between the rank and the click rate of search ads}

Insufficient scientific evidence exists about why the top-ranked article is the first read and where the consumer's attention focuses on a web screen of a newspaper article for the first time.

Kim and Park (2005) studied attention given by the human instinct by using Think Aloud methods. This study monitored eye movements of subjects that were looking at a newspaper article displayed on the screen. As a result, if there are many articles in the top left, it becomes the starting point of the path for the users' eyes.

There is also no scientific evidence regarding why consumers click the first ranked ads in the top left corner of the screen first and often, when the search ads are ordered. A very common idea is that people will search the information retrieved first, and then move downward. However, according to the study demonstrating the correlation between the ranking of search ads and the CTR, we can know that the ranking has greater impact on CTR.

Atlas surveyed Google and Overture which lead the world's search ad market, and reported the change in CTR based on the search ad ranking. Potential CTR data were derived from the number of exposers and clicks, and the high ranked search ads were significant for both Google and Overture. 
As shown in Figure 2, Google's top priority and second priority ads were separated by 405 clicks, the difference between second position and third position was about $20 \%$, and then a modest difference occurred after the third place. In the case of Overture, there was approximately a $22 \%$ difference between the first position and second position, and also the difference between positions 2 through 5 was noticeable. Therefore, this report concluded that advertisers ranked their ads keywords in the top of the list, in order to maximize the effectiveness of search ads (Atlas DMT, 2004).

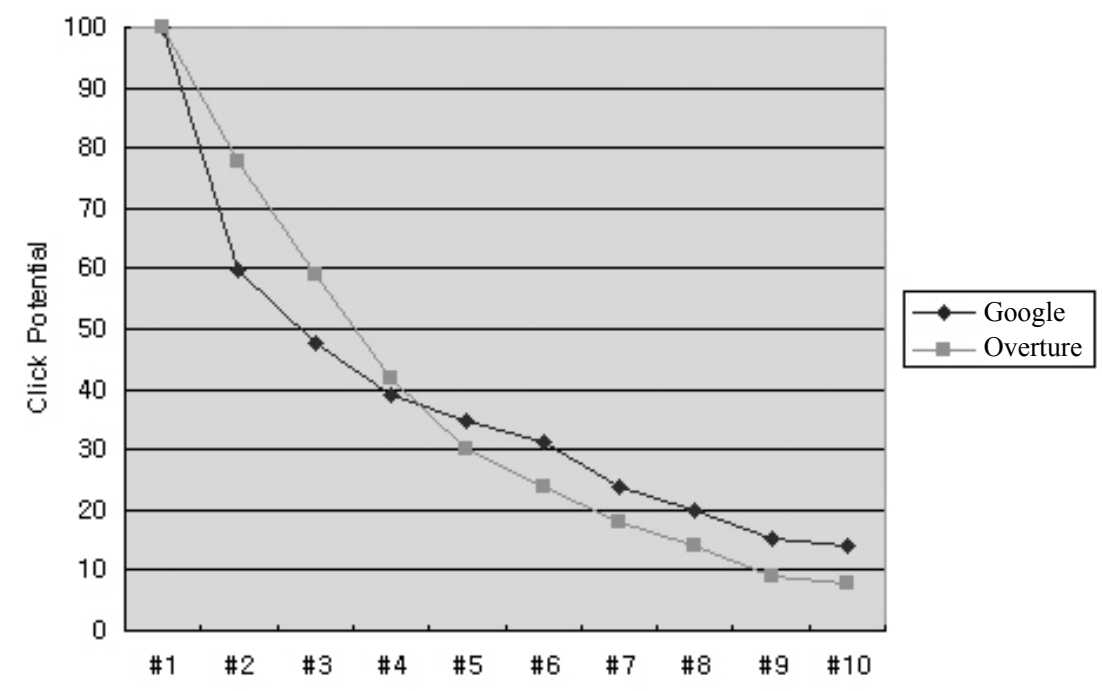

Fig. 2. CTR's change according to the rank of search ads Source: Atlas DMT 2004

A similar result to the above study was shown in Korean studies. In the experimental study about click action based on the search ads' location, the ads appearing at the top of a Web page have a higher CTR (Kim, Kim, \& Kim, 2002). According to the KoreanClick's survey research, the website users consider rank the most important criteria, followed by a faithful description, a prominent heading, and then an awareness of the site (KoreanClick, 2005).

\section{Research Design and Research Method}

\subsection{Research Design}

\subsubsection{Site selection and the size of parameters and samples}

Company $\mathrm{S}^{\prime}$ marketing site, which is advertised via search ads, was selected as the target site. The full data set for this study is the total number of exposures of search ads per year which is brought up by a keyword such 'IDC', 'hosting', and 'colocation'. The sample set is the number of the keywords' exposures within a six-week period. 


\subsubsection{Categories of keywords and their definition}

The selected keywords for this study are the keywords used in situations where the consumer's involvement is high, those primarily used by practitioners in the field of IT or SOHO businessmen in an internet-related field. These keywords and their categories are shown in Table 1.

Table 1. Categories of keyword

\begin{tabular}{llll}
\hline $\begin{array}{l}\text { Keyword } \\
\text { Categories }\end{array}$ & IDC & Hosting & Colocation \\
\hline extended & 11 variations including & 35 variations including & 9 variations including, \\
keyword & IDC Center, & Hosing, Server Hosting, & Colocation, \\
& IDC, IDC Server, Moving & Hosting company, Server, & Colocation specialist, etc. \\
& IDC, etc. & Server Leasing, etc. & \\
\hline
\end{tabular}

\subsubsection{Definitions of types of search ads messages}

Advertising messages were specified for the categories of three keywords. In order to investigate the difference between consumers' CTR based on the ad messages, two types were defined. The phrases for type A were specified to emphasize the reality and quality and selected primarily in the situation of high involvement. The phrases for type B were chosen to highlight events such as price discounts and free services in the situations of low involvement.

Table 2. The definitions of message types of search ads

\begin{tabular}{|c|c|c|}
\hline Division & Topic & Advertisement message \\
\hline \multirow[t]{3}{*}{ Type A } & Hosting & high-quality server hosting provided by company $\mathrm{S}$ \\
\hline & IDC & $\begin{array}{l}\text { high-quality managed hosting, server hosting, colocation of company S's } \\
\text { IDC }\end{array}$ \\
\hline & Colocation & $\begin{array}{l}\text { state-of-the-art IDC based on colocation services, dedicated server space } \\
\text { rental, high-speed Internet backbone connection, } 24 \text {-hour service management }\end{array}$ \\
\hline \multirow[t]{3}{*}{ Type B } & Hosting & $\begin{array}{l}\text { HP dual-core server ( } 900,000 \text { won), additional quad-core equipment disk, } \\
\text { free monitoring service }\end{array}$ \\
\hline & IDC & $\begin{array}{l}\text { special price for quad-core server, special price for HP dual-core server, } \\
\text { additional disk, free monitoring service }\end{array}$ \\
\hline & Colocation & $\begin{array}{l}\text { dedicated server space rental, special offers server, additional disk, free } \\
\text { monitoring service }\end{array}$ \\
\hline
\end{tabular}

* Type A: Emphasizing reliability and quality

* Type B: Emphasizing events such as price discounts, free services, etc.

\subsubsection{Perform search ads and data acquisition}

In order to investigate the effects that advertising message types and search ads ranks have on the CTR, ads grouped by message type and by ads ranks (rank 1, rank 3, and rank 5) were exposed six times (See Table 3). As a result, the data collected included the number of ads exposed to the portal, and the number of clicks on S's website search ads meaning the number of consumers visiting the S's website. 
Table 3. Data about advertising messages and rank

\begin{tabular}{lll}
\hline Type of ads & Duration of ad exposure & Data collection \\
\hline Type A, Rank 1 & November, $1^{\text {st }}$ week & Data of five days except for Saturdays and \\
Type A, Rank 3 & November, $2^{\text {nd }}$ week & \\
Type A, Rank 5 & November, $3^{\text {rd }}$ week & \\
Type B, Rank 1 & November, $4^{\text {th }}$ week & \\
Type B, Rank 3 & November, $5^{\text {th }}$ week & \\
Type B, Rank 5 & December, $1^{\text {st }}$ week & \\
\hline
\end{tabular}

\subsection{Procedures and methods of data collection}

The method and process of data collection for this study are as follows Figure 3.

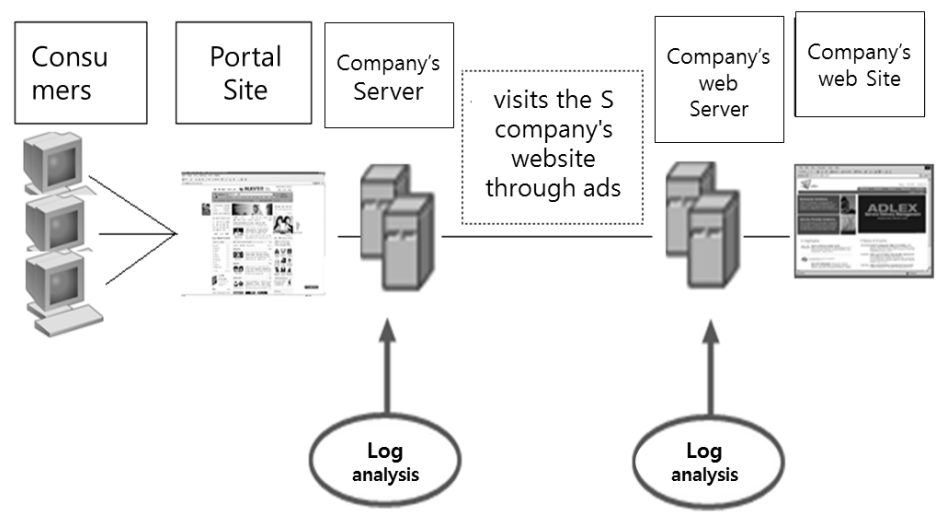

Fig. 3. Data acquisition procedure of search ads

(1) The consumers access the portal site for using the search ads

(2) The related companies' advertisement is exposed to the portal site if any consumers search for keywords such as 'IDC', 'hosting', or 'colocation' at the portal site. At this time, the number of ads exposed is recorded to the ads company's server for log analysis.

(3) The consumer visits the S company's website by clicking the $\mathrm{S}$ company's search ads among the exposed advertisements. At this point, the number of clicks on search ads is recorded to the ads company's server for log analysis.

(4) The consumer searches for his desired information on the S company's website. At this point, connection information and access paths of the consumer are recorded to the ads company's server for $\log$ analysis.

(5) The search advertising company provided the number of expose / click / CTR / CPC advertising about S company as daily / weekly / monthly reports.

(6) The researcher verified the accuracy of the data from the search advertising company by comparing it to the data from S's own log analysis tools. 


\section{International Journal of Knowledge Content Development \& Technology Vol.1, No.1, 7-24 (June, 2011)}

\subsection{Data extracted for statistical analysis}

In order to quantitatively analyze statistical differences in the number of website visitors before and after performing search ads, we extracted from S company's web log analysis server data from October, when search ads were not present and data from November when search ads were present (See Table 4).

Table 4. The number of website visitors before and after search ads

\begin{tabular}{|c|c|c|c|c|c|c|c|c|c|c|c|c|c|c|c|}
\hline & \multicolumn{15}{|c|}{ Daily website visitors } \\
\hline & 1 & 2 & 3 & 4 & 5 & 6 & 7 & 8 & 9 & 10 & 11 & 12 & 13 & 14 & 15 \\
\hline Oct. & 212 & 226 & 207 & 151 & 162 & 140 & 133 & 148 & 191 & 180 & 201 & 158 & 133 & 126 & 146 \\
\hline \multirow[t]{2}{*}{ Nov. } & 1,901 & 1,676 & 1,119 & 1,148 & 2,090 & 1,851 & 1,678 & 1,644 & 1,464 & 1,010 & 1,092 & 2,015 & 2,101 & 2,007 & 1,803 \\
\hline & 16 & 17 & 18 & 19 & 20 & 21 & 22 & 23 & 24 & 25 & 26 & 27 & 28 & 29 & 30 \\
\hline Oct. & 175 & 197 & 169 & 191 & 140 & 112 & 141 & 127 & 169 & 143 & 172 & 161 & 95 & 165 & 183 \\
\hline Nov. & 1,788 & 1,208 & 1,151 & 2,054 & 1,908 & 1,899 & 1,696 & 1,713 & 1,085 & 1,104 & 2,165 & 2,008 & 1,926 & 1,958 & 1,570 \\
\hline
\end{tabular}

Table 5 shows data about the advertising message types and ads ranks.

Table 5. Types of advertising messages and advertising rank data

\begin{tabular}{llllll}
\hline $\begin{array}{l}\text { Type of } \\
\text { message }\end{array}$ & Rank & Ad duration of exposure & Impressions & Click Count & CTR(\%) \\
\hline A & 1 & The $1^{\text {st }}$ week of November & 9,838 & 80 & 0.81 \\
& 2 & The $2^{\text {nd }}$ week of November & 8,606 & 47 & 0.55 \\
& 3 & The $3^{\text {rd }}$ week of November & 9,054 & 41 & 0.45 \\
B & 1 & The $4^{\text {th }}$ week of November & 9,305 & 49 & 0.53 \\
& 2 & The $5^{\text {th }}$ week of November & 10,908 & 36 & 0.33 \\
& Position 3 & The $1^{\text {st }}$ week of December & 10,059 & 24 & 0.24 \\
\hline
\end{tabular}

* Impressions: the number of S's ad impressions exposed to the entire portal during advertising period

* Click count: The number of the consumers' visits to the S company's website by clicking the S company's advertising

* CTR: Click Through Rate $=$ Click count/Impressions

\subsection{Statistical analysis methods}

In this paper, I used 2-Sample-t as quantitative statistical analysis methods to analyze changes in the number of website visitors before and after performing the search ads. To analyze the difference of CTR based on the type of advertising messages and ad position, 2-Proportion and Chi-Square analysis were used. After setting the null hypothesis and alternative hypothesis, I verified the hypothesis in the trust level of 1- $\alpha: 95 \%$, significance level of a: $5 \%$ by comparing the P-value. The Minitab 14 Statistical Software was used for the statistical analysis of the experiment. 


\subsection{Hypothesis}

According to the theory of involvement, consumers will click on the ads with messages emphasizing quality and reliability by increasing the involvement, when they search for more expensive items or are in searching situations requiring a lot of cognitive effort. On the other hand, consumers will click on the ads with messages highlighting price discounts or events at a low level of the involvement, when searching for cheaper items or searching for items that can be selected without having to worry (Petty \& Cacioppo, 1986; Hoffman \& Novak, 1996; Yoon \& Kim, 2002). In addition, the domestic and international empirical studies reported that CTR differs according to the rank of ads (Kim, Kim, \& Kim, 2002; Kim \& Park, 2005; Atlas DMT, 2004; KoreanClick, 2005). Therefore, I set the following three central hypotheses to verify the above theories by using actual case data.

$\mathrm{H}$ 1: The conducting of search advertising would affect consumers' website visits.

H 2: The message type of search advertising would affect consumers' website visits.

$\mathrm{H}$ 3: The ranking of search advertising would affect consumers' website visits.

\section{Results}

\subsection{Verification of the change of website visits based on conducting the search ads}

As shown in Table 6, the S company's website visits increased tenfold after conducting search advertising. I carried out the 2-Sample $\mathrm{T}$ analysis to verify the statistical significance of this difference by using the data in Table 6 and setting the hypothesis.

- Null Hypothesis: The execution of search advertising will not have an impact on website visitors.

- Alternative Hypothesis: The execution of search advertising will have an impact on website visitors.

P-value $=0.001$ in test results is less than 0.05 , and so the null hypothesis is rejected and alternative hypothesis is adopted. Therefore, it can be concluded that the company's website visits would be increased if the company conducted the search ads.

\subsection{Verification of the change of website visits based on the type of search advertising messages}

\subsubsection{Comparing CTR of type $A$ and $B$ of ad's message}

I carried out the 2-Proportion analysis to verify the statistical significance of the difference to find out whether the message type of search ads affects website visits or not, by setting the hypothesis and using the data in Table 6.

- Null Hypothesis: The message type of search advertising would not affect the consumers' website visits.

- Alternative Hypothesis: The message type of search advertising would affect the consumers' website visits. 
J. S. Son
International Journal of Knowledge Content Development \& Technology Vol.1, No.1, 7-24 (June, 2011)

Table 6. Comparing CTR of type A and B of ads messages

\begin{tabular}{llllllll}
\hline Type & Rank & Exposure & Clicks & CTR & relative values & P-value & test results \\
\hline A & $1,3,5$ & 27,498 & 168 & $0.61 \%$ & 100 & 0.000 & Significant \\
B & $1,3,5$ & 30,272 & 109 & $0.36 \%$ & 59 & & \\
\hline
\end{tabular}

$\mathrm{P}$-value $=0.000$ in test results is less than 0.05 . Therefore the null hypothesis is rejected and alternative hypothesis is adopted. Based on the findings, the appropriate choice of advertising message type influences the consumers' website visits. Therefore it could be concluded that the message type of search ads for emphasizing the reliability and quality is valid in order to increase the company's website visits from the consumers who search the advertising with keywords of high involvement.

\subsubsection{Comparing the CTR of $A$ and $B$ types of advertising messages (1-ranked search ads)}

I carried out the 2-Proportion analysis by setting the hypothesis and using the data in Table 7 ato verify statistical significance of the difference to find out whether the message type of search ads affects website visits or not in the conditions of 1-ranked search ads.

- Null Hypothesis: The message type of search advertising would not affect the consumers' website visits in the conditions of 1-ranked search ads.

- Alternative Hypothesis: The message type of search advertising would affect the consumers' website visits in the conditions of 1-ranked search ads.

Table 7. Comparing the CTR of A and B types of advertising messages ( $1^{\text {st }}$ ranked search ads)

\begin{tabular}{llllllll}
\hline Type & Rank & Exposure & Clicks & CTR & relative values & P-value & test results \\
\hline A & 1 & 9,838 & 80 & $0.81 \%$ & 100 & 0.015 & Significant \\
B & 1 & 9,305 & 49 & $0.53 \%$ & 65 & & \\
\hline
\end{tabular}

Test results showed that the P-value is 0.015 , so significantly smaller than 0.05 to reject the Null Hypothesis and to choose the Alternative Hypothesis. Therefore, in the conditions of 1-ranked search ads, it could also be concluded that the message type of search ads for emphasizing reliability and quality is valid in order to increase the company's website visits from the consumers who search the advertising with keywords of high involvement.

\subsubsection{Comparing the CTR of $A$ and $B$ types of advertising messages ( $3^{\text {rd }}$ ranked search ads)}

I tried to find out whether the message type of search ads affects website visits or not in the conditions of $3^{\text {rd }}$ ranked search ads and carried out the 2-Proportion analysis by setting the hypothesis and using the data in Table 8 to verify statistical significance of the difference.

Table 8. Comparing the CTR of $\mathrm{A}$ and $\mathrm{B}$ types of advertising messages ( $3^{\text {rd }}$ ranked search ads)

\begin{tabular}{llllllll}
\hline Type & Rank & Exposure & Clicks & CTR & relative values & P-value & test results \\
\hline A & 3 & 8,606 & 47 & $0.55 \%$ & 100 & 0.025 & Significant \\
B & 3 & 10,908 & 36 & $0.33 \%$ & 60 & & \\
\hline
\end{tabular}


Test results showed that the P-value is 0.025 , so significantly smaller than the 0.05 to reject the Null Hypothesis and to choose the Alternative Hypothesis. Therefore, in the conditions of 3-ranked search ads, it could also be concluded that the message type of search ads for emphasizing the reliability and quality is valid in order to increase the company's website visits from the consumers who search the advertising with keywords of high involvement.

\subsubsection{Comparing the CTR of $A$ and $B$ types of advertising messages (5 $5^{\text {th }}$ ranked search ads)}

I tried to find out whether the message type of search ads affects website visits or not in the conditions of $5^{\text {th }}$ ranked search ads and carried out the 2-Proportion analysis by setting the hypothesis and using the data in Table 9 to verify statistical significance of the difference.

Table 9. Comparing the CTR of A and B types of advertising messages ( $5^{\text {th }}$ ranked search ads)

\begin{tabular}{llllllll}
\hline Type & Rank & Exposure & Clicks & CTR & relative values & P-value & test results \\
\hline A & 5 & 9,054 & 41 & $0.45 \%$ & 100 & 0.024 & Significant \\
B & 5 & 10,059 & 24 & $0.24 \%$ & 53 & & \\
\hline
\end{tabular}

Test results showed that the P-value is 0.024 , so significantly smaller than the 0.05 to reject the Null Hypothesis and to choose the Alternative Hypothesis. Therefore, in the conditions of 1-ranked search ads, it could also be concluded that the message type of search ads for emphasizing the reliability and quality is valid in order to increase the company's website visits from the consumer who search the advertising with keywords of high involvement.

\subsection{Verification of the change of website visits based on the rank of search advertising}

\subsubsection{Comparing the CTR of Search Ad Rank 1, 3, 5}

I tried to find out whether the rank of search ads that appeared when the consumer searched with keywords such as 'IDC', 'Hosting', and 'colocation' would affect their website visits, and carried out the 2-Proportion analysis by setting the hypothesis and using the data in Table 10 to verify the statistical significance of the difference.

- Null Hypothesis: The rank of search ads would not affect website visits.

- Alternative Hypothesis: The rank of search ads would affect website visits.

Table 10. Comparing the CTR of Search Ad Rank 1, 3, 5

\begin{tabular}{llllllll}
\hline Type & Rank & Exposure & Clicks & CTR & relative values & P-value & test results \\
\hline A,B & Rank 1 & 19,143 & 129 & $0.67 \%$ & 100 & 0.000 & Significant \\
A,B & Rank 3 & 19,514 & 83 & $0.43 \%$ & 63 & & \\
A,B & Rank 5 & 19,113 & 65 & $0.34 \%$ & 50 & & \\
\hline
\end{tabular}

The test results showed that the P-value is 0.000 , so significantly smaller than the 0.05 needed to reject the Null Hypothesis and to choose the Alternative Hypothesis. Therefore, it could be concluded that the rank of search ads affects the company's website visits. Namely, in order to 
increase the company's website visits, search ads should be ranked as highly as possible.

\subsubsection{Comparing the CTR of Search Ad Rank 1, 3, 5 (Search Ads Message Type A)}

In the conditions of search ads message type A that emphasizes the quality and reliability, I tried to find out the difference of website visits at Search Ad Rank 1, 3, 5, and carried out the Chi-Square test by setting the hypothesis and using the data in Table 11 to verify statistical significance of the difference.

- Null Hypothesis: In the conditions of search ads message type A, the rank of search ads would not affect website visits.

- Alternative Hypothesis: In the conditions of search ads message type A, the rank of search ads would affect website visits.

Table 11. Comparing the CTR of Search Ad Rank 1, 3, 5 (Search Ads Message Type A)

\begin{tabular}{llllllll}
\hline Type & Rank & Exposure & Clicks & CTR & relative values & P-value & test results \\
\hline A & 1 & 9,838 & 80 & $0.81 \%$ & 100 & 0.004 & Significant \\
A & 3 & 8,606 & 47 & $0.55 \%$ & 67 & & \\
A & 5 & 9,054 & 41 & $0.45 \%$ & 56 & & \\
\hline
\end{tabular}

The test results showed that the P-value is 0.004 , so significantly smaller than the 0.05 needed to reject the Null Hypothesis and to choose the Alternative Hypothesis. Therefore, it could be said that the rank of search ads affects company S's website visits in the conditions of search ads message type A, which emphasizes the quality and reliability.

However, in the same conditions of message type A, there was a statistically significant difference between 1 ranking and 3 or 5 rankings, but there was no statistically significant difference between 3 ranking and 5 ranking.

\subsubsection{Comparing the CTR of Search Ad Rank 1, 3, 5 (Message Type B)}

In the conditions of message type $B$, which emphasizes price discount and events, I tried to find out the difference of website visits at Search Ad Rank 1, 3, 5, and carried out the Chi-Square test by using the data in Table 12 to verify statistical significance of the difference.

Table 12. Comparing the CTR of Search Ad Rank 1, 3, 5 (Message Type B)

\begin{tabular}{llllllll}
\hline Type & Rank & Exposure & Clicks & CTR & relative values & P-value & test results \\
\hline B & 1 & 9,305 & 49 & $0.53 \%$ & 100 & 0.003 & Significant \\
B & 3 & 10,908 & 36 & $0.33 \%$ & 63 & & \\
B & 5 & 10,059 & 24 & $0.24 \%$ & 45 & & \\
\hline
\end{tabular}

The test results showed that the P-value is 0.003 , so significantly smaller than the 0.05 needed to reject the Null Hypothesis and to choose the Alternative Hypothesis. Therefore, it could be said that the rank of search ads affects the company S's website visits in the conditions of the message type $\mathrm{B}$, which emphasizes the price discount and events. 
However, in the same conditions of the message type B, there was a statistically significant difference between $1^{\text {st }}$ ranking and $3^{\text {rd }}$ or $5^{\text {th }}$ rankings, but there was no statistically significant difference between $3^{\text {rd }}$ ranking and $5^{\text {th }}$ ranking $(\mathrm{P}$-value $=0.213>0.05)$.

\subsection{Verification of the difference of website visits based on the combination of message type and rank}

I tried to find out which factors heavily affect the consumer's website visits by statistically comparing and analyzing the various combinations of $t$ search ads message type and rank.

\subsection{1. (Message Type A, Rank 3) and (Message Type B, Rank 1)}

I tried to analyze the statistical differences of website visits between when the search ads are exposed in the $3^{\text {rd }}$ ranked position with message type $\mathrm{A}$ and when the search ads are exposed in $1^{\text {st }}$ ranked position with message type $\mathrm{B}$, and carried out the 2-Proportion analysis by using the data in Table 13 to verify statistical significance of the difference.

Table 13. Comparison between the combination: (Type A, Rank 3) and (Type B, Rank 1)

\begin{tabular}{llllllll}
\hline Type & Rank & Exposure & Clicks & CTR & relative values & P-value & test results \\
\hline A & 3 & 8,606 & 47 & $0.55 \%$ & 100 & 0.858 & Not Significant \\
B & Rank 1 & 9,305 & 49 & $0.53 \%$ & 96 & & \\
\hline
\end{tabular}

According to the test results, there was no significant difference between the two conditions because the P-value $=0.858$ is significantly larger than 0.05 .

\subsection{2. (Message Type A, Rank 5) and (Message Type B, Rank 1)}

In the conditions of Message Type A, Rank 5 and Message Type B, Rank 1, 'Not significant' results were derived with $\mathrm{P}$-value $=0.474$. After all, if the company uses message type $\mathrm{B}$, even if the ads rank is reduced to five, the company could get a similar effect with the case of the message type B and the $1^{\text {st }}$ ranked search ads. In other words, we can say that the consumers respond to the content of advertising messages rather than the rank of ads in conditions of high involvement.

Table 14. Comparison between the combination: (Type A, Rank 5) and (Type B, Rank 1)

\begin{tabular}{llllllll}
\hline Type & Rank & Exposure & Clicks & CTR & relative values & P-value & test results \\
\hline A & 5 & 9,054 & 41 & $0.45 \%$ & 100 & 0.474 & Not Significant \\
B & 1 & 9,305 & 49 & $0.53 \%$ & 116 & & \\
\hline
\end{tabular}




\section{Analysis of the Relative Effect of a Combination of Advertising}

\subsection{Analysis Matrix of CTR based on the combination of ads message and rank}

The above test results for the combination of the message type and rank of search ads can be summarized as the matrix of effect analysis as shown in the following Table 15. The consumer in the circumstance of high involvement showed a very high CTR when the message type of search ads is oriented to quality and reliability. In brief, there was no statistically significant difference between 'Type A in Rank 5' to pay low cost and 'Type B in Rank 1' to pay high cost.

Table 15. Analysis Matrix of CTR based on the combination of Ad type and rank

\begin{tabular}{llll}
\hline Combination of Ad & (Type B, $1^{\text {st }}$ Rank) & (Type B, $3^{\text {rd }}$ Rank) & (Type B, $5^{\text {th }}$ Rank) \\
\hline Type A, $1^{\text {st }}$ Rank & $>$ & $>$ & $>$ \\
& Result: Significant & Result: Significant & Result: Significant \\
Type A, $3^{\text {rd }}$ Rank & $=$ & $>$ & $>$ \\
& Result: Not Significant & Result: Significant & Result: Significant \\
Type A, 5 $5^{\text {th }}$ Rank & $=$ & $=$ & $>$ \\
& Result: Not Significant & 결과: Not Significant & Result: Significant \\
\hline
\end{tabular}

* Significant: There is significant difference in the number of website visits.

* $>$ : The combination of advertising representing a high CTR (Website visits)

\subsection{Cost-effectiveness by a combination of advertising}

The cost-effectiveness and efficiency of the combination of search ads is shown in the following Table 16.

Table 16. Cost-effectiveness of a combination of advertising

\begin{tabular}{lllll}
\hline Type/Rank & Exposure & Clicks & CTR & $\begin{array}{l}\text { CTR rank per 100,000 } \\
\text { won* of advertising costs }\end{array}$ \\
\hline Type A, $1^{\text {st }}$ Rank & 9,838 & 80 & $0.81 \%$ & 5 \\
Type A, 3 $3^{\text {rd }}$ Rank & 8,606 & 47 & $0.55 \%$ & 1 \\
Type A, 5 $5^{\text {th }}$ Rank & 9,054 & 41 & $0.45 \%$ & 2 \\
Type B, 1 $1^{\text {st }}$ Rank & 9,305 & 49 & $0.53 \%$ & 4 \\
Tyep B, 3 $3^{\text {rd }}$ Rank & 10,908 & 36 & $0.33 \%$ & 5 \\
Type B, 5 $5^{\text {th }}$ Rank & 10,059 & 24 & $0.24 \%$ & 2 \\
\hline
\end{tabular}

* 100,000 won is about 100 dollar.

By referring to company S's case shown in Table 16, I can present the model for executing search ads that is appropriate for service products of high involvement. In certain circumstances when the advertising budget was set, search ads budget may be used depending on the scheduling strategy within a certain period of time. In the short term, they might be uniformly distributed throughout the year on an ongoing basis in the form of continuous advertising. 
The most effective advertising methods to increase the consumers' website visits in a short term is 'Type A, Rank 1' because CTR is the highest. On the other hand, the most effective advertising methods for conducting effective long-term advertising is 'Type A, Rank 3' which is the highest in the CTR per 100,000 won (about \$100).

\section{Discussion and Conclusion}

\subsection{Findings}

Internet search advertising is growing rapidly as a important section of the internet advertising market, showing the limitless potential for growth by applying the marketing techniques and the advanced technology of the future.

This study tried to verify the effect of company website visits when internet search advertising on the portal is exposed. To do this, 'IDC', 'hosting', 'colocation', which have high involvement in the IT field, were exposed to search advertising, the effect of the message type and rank of search ads was analyzed through the CTR. The results are as follows:

First, it was shown that conducting search ads increases the company's website visits very significantly. There was a difference of more than 10 times after adding search ads compared to before adding it.

Second, in the situations of high involvement, there was a large difference in CTR when the consumer came in contact with the keywords such as 'IDC', 'hosting', 'colocation'. The ads messages that emphasize the quality and reliability showed a higher CTR than the ads message that highlight price discounts and events.

Third, the search ads' ranking exposed to consumers affected the ad CTR, therefore the CTR of ads showed a statistically significant difference between the ads of Rank 1 and the ads Rank 3 or 5 . The results of these types was the same both when the advertising message that emphasizes the quality and reliability was selected and when the advertising message that highlights price discounts and events was selected. Meanwhile, this study analyzed statistically various combinations of message type and rank of ads, to find out which factor affects the company's website visits. The results are as follows:

In situations of high involvement where the consumers shows a greater response to quality and reliability of the type of advertising message rather than the rank, the company's message type oriented on quality and reliability showed a very high CTR. There was an $8 \%$ difference between the case of cheap costs that the Ads message to emphasize the quality and reliability was exposed in the $5^{\text {th }}$ rank and the case of high costs that the ads message to highlight the price discount and events was exposed in the $1^{\text {st }}$ rank, but statistically showed no significant differences. The search ads' CPC (Cost per Click) is determined depending on the rank of ads. Therefore, we may get the same effect with the high cost of $1^{\text {st }}$ ranked ads which is not suitable for all situations, by exposing the $5^{\text {th }}$ ranked ads message appropriate for any situation.

The advertisers should be forced to execute the most cost-effective search ads within the scope 
of budget. For this, the first step for the purpose of advertising is to have a well-established strategy for scheduling. Depending on the scheduling strategy that focuses on the short-term, CTR may continue to increase.

According to my research, if one wants to increase ads' effectiveness by fully focusing on the short-term, the $1^{\text {st }}$ ranked advertising with high price will be more effective and show a high CTR. On the other hand, if one wants to increase the company's awareness in a continuous form, the $3^{\text {rd }}$ ranked advertising with the appropriate price will be more cost-effective and show the highest CTR per 100,000 won.

Advertisers have to well define the message type of search ads appropriate for the situation, and monitor the reaction of consumers periodically. At the same time, they should be adjusting the ads' rank considering the budget and the ad's CTR. If these efforts are continuous, the company can achieve the purpose of search ads as much as possible.

\subsection{Implications}

This study has overcome the limitations of the previous study that the experimental conditions differed from the actual situation, by collecting and analyzing the actual data when the consumers connected from the portal site to the company's website through the search ads. In this study, subjects were exposed to the experiment without being aware of any intent of the effectiveness of advertising on the Internet search.

This study verified that the search ads of high involvement such as 'IDC', 'hosting', 'collocation' showed high advertising effectiveness when exposed as the ads message to highlight the quality and reliability. In addition, it was verified that the consumer of high involvement considers the message type of ads more seriously than the rank of ads. It implies that selecting the better search advertising message is more important than exposing at an expensive high rank. Therefore, these findings would be very meaningful to advertisers who provide telecommunication services.

This study also presented the most cost-effective search advertising model that the advertisers in the field of information and communications service may experience through the matrix analysis of specific numerical cases. In other words, CTRs per advertising costs unit were calculated by the matrix of 'exposers', 'clicks', 'CTR', and 'CPC' created in accordance with the message type and rank of search ads. It would be a helpful model that the advertisers could adopt the most cost-effective ads depending on the scheduling strategy.

\subsection{Limitations and Further Research}

Even though this study analyzed the effectiveness of internet search advertising based on real data, it has some limitations and future research is needed to compensate for this.

First, this study conducted a case analysis by targeting only search ads in IT services in the situations of high involvement. Even though it was verified statistically that the consumer's reaction in the situation of high involvement was consistent with the theory, the consumer's reaction in low involvement are needed to test in further studies. 
Second, there are daily and monthly fluctuations in terms of the exposures and clicks of search ads. Even though this study got rid of the variable of the daily fluctuation by using data for weeks of November and December, It could not eliminate the impact of monthly variations. To overcome this limitation, it is necessary to set the data collection at least annually.

Third, CTRs could appear differently according to consumers' awareness of companies which are exposed at the same time. Nevertheless, this study doesn't consider or include the impact of brand awareness. Therefore follow-up studies to investigate the impact of brand awareness are needed.

\section{Acknowledgements}

This work was rewritten based on a Master's thesis. The author would like to thank Patricia Ladd for editing this paper into fluent American English.

\section{References}

Atlas DMT. (2004). How Search Engine Rank Impacts Traffic, starts off by asking the provocative question: "What does being number one in search really mean to your business?". Atlas DMT.

Hoffman, Donna L. and Thomas Novak. (1996). "Marketing in Hypermedia Computer-Mediated Environments: Conceptual Foundations." Journal of Marketing, 60(July), 50-68.

Jordan McCollum. (2007).

$<$ http://www.marketingpilgrim.com/2007/08/eyetracking-shows-web-audience-ignores-ads.ht $\mathrm{ml}>$ [2007.11.20].

Kim, Bun-tae and Jong-Myeung Kim. (2003). A Study on the Online Advertising Effectiveness of a Product Fit, an Involvement toward the Website and a Click Act. Marketing Management Research, 8(3), 55-81.

Kim, Jaehwi, Jiho Kim, \& Yonghwan Kim. (2002). A study on advertising effectiveness of internet portal site. Marketing Research, 13(3), 91-109.

Kim, Jiho \& Jaehwi Kim. (2003). Influences of stimulation and avoidance of internet advertising on advertising effectiveness. Marketing Research, 14(3), 165-190.

Kim, Jiho, Jaehwi Kim, Hacheol Park, \& Janghan Lee. (2006). A study on the effects of selective attention factors of internet advertising utilizing Eye tracker. Marketing Research, (72), 31-58.

Kim, Jongwoo, \& Seungyeop Lee. (2001). A study on the effect of internet advertising by product involvement levels, website types and banner advertisement types. Journal of Global Academy of Marketing Science, 7, 105-124.

Kim, Seunggyu. (2003). A study on Internet search advertising effectiveness analysis based on the type of research. Korea University, Master's paper.

Kim, teayong, \& Jeayoung Park. (2000). Verification a notificaiton of the newspaper article and the path of eyes by using Think Aloud. Korea Press Gazette, 49(4), 87-101. 
KoreanClick. (2005). The necessity of objective data for Search Engine. 2005 Search Ad Marketing Conference Seminar paper.

Lee, Jongchun, Hyewon Lee, \& Gyohong Jin. (2003). Implementation of new internet advertising method using text keyword. Information and Communication Research, 4,189-196.

Lee, Juhyeon, \& Younggyun Choi. (2002). The effects of perceived interactivity on users' involvement, attitude, and revisiting intention on a search engine Web Site. Marketing Research, 13(5), 223-248.

Petty, R. E. and J. T. Cacioppo. (1986). Communication and Persuasion: Central and Peripheral Route to Attitude Change. NY: springer-Verlag.

Sandra E. Moriarty. (1991). Creative Advertising: Theory and Practice. New Jersey: Prentice-Hall, Inc.

Yoon, Seongjun, \& Juho Kim. (2002). Banner ads are effective?: Focusing forms and contents of advertising. Marketing Research, 13(3), 141-162. 